\title{
A Comparison of Theory of Mind and Empathy in Male and Female Students at University of Welfare and Rehabilitation Sciences in Tehran
}

\author{
Ebrahim Soltani Azemat ${ }^{1}$, Abolfazl Mohammadian ${ }^{1 *}$, Maryam Kamie ${ }^{1}$, Maryam Jebreeili ${ }^{2}$, Behrooz Doolatshahi ${ }^{1}$ \\ ${ }^{1}$ Department of Clinical Psychology, University of Social Welfare and Rehabilitation Sciences, Tehran, Iran \\ ${ }^{2}$ Department of Psychology, Faculty of Education and Psychology, University of Tabriz, Tabriz, Iran
}

\section{A BSTRACT}

Introduction: Empathy and theory of mind, capacities that are essential for human communication, are abilities which highly correlated to each other. Several studies have shown that women outperform men regarding empathy. However, using theory of mind did not confirm these results. The aim of the present study was to compare theory of mind and empathy in male and female students at University of Welfare and Rehabilitation Sciences in Tehran. Materials and Methods: The research was a descriptive-comparative study. One hundred undergraduate students, 50 males and 50 females (due to the population size by using the Cochran formula), from different rehabilitation majors took part in this study. They were selected by non-probability quota sampling. Davis empathy questionnaire and Baron Cohen Eyes Test were used for this evaluation. Results: The findings showed that women outperformed men significantly with regard to empathy test scores. However, there was not a statistically significant difference between men and women in theory of mind test scores. Conclusion: One possible reason for different findings between two methods used in this study may be the self-report nature of sympathy questionnaire. Women are expected to be more empathic in society and this can affect their responses. Theory of mind test emphasizes on more complex mind states and examines empathy in an indirect way could be a more reliable test.

\section{Key words:}

1. Empathy

2. Theory of Mind

3. Rehabilitation

* Corresponding Author: Abolfazl Mohammadian

E-mail:abolfazl796@gmail.com 


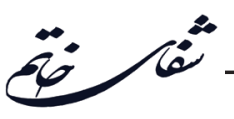

مقايسةُ نظريةٌ ذهن و همدلى در دانشجويان يسر و دختر در دانشعاه علوم بهزيستى و توانبخشى تهران

ابر اهيم سلطانى عظمت'، ابوالفضل محمديان'"، مريم كامى'،مريم جبرئيلى '،بهروز دولتشاهى'

'اَّروه روانشناسى بالينى، دانشكاه علوم بهزيستى و توانبخشى، تهران، ايران

بكروه روانشناسى، دانشكده علوم تربيتى وروانشناسى، دانشكاه تبريز، تبريز، ايران

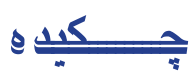

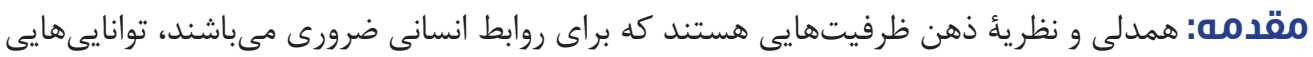

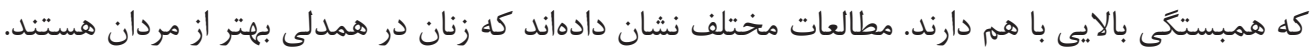

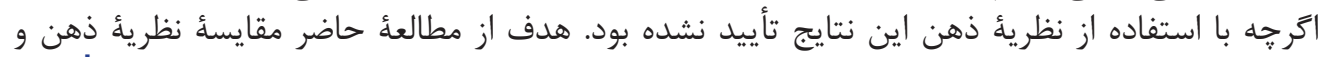

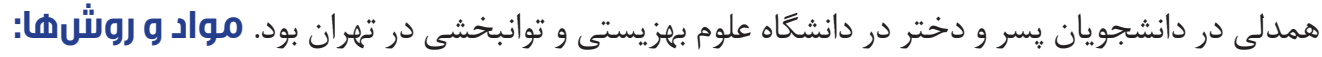

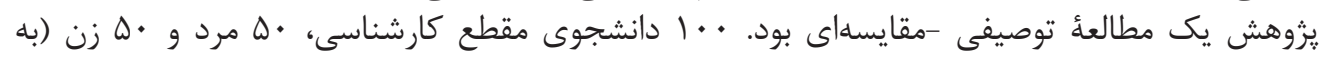

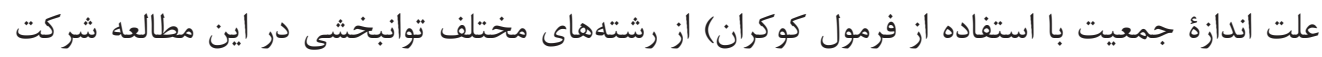

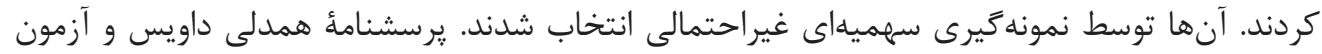

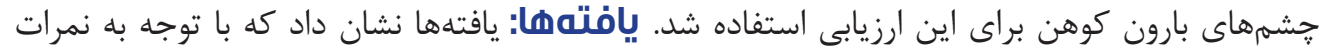

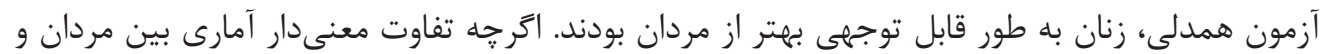

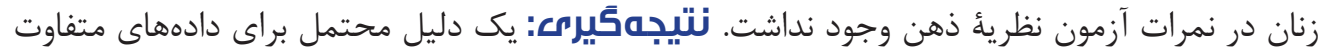

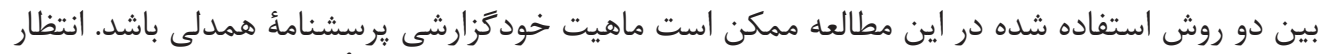

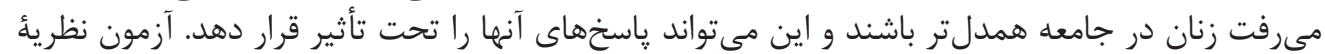

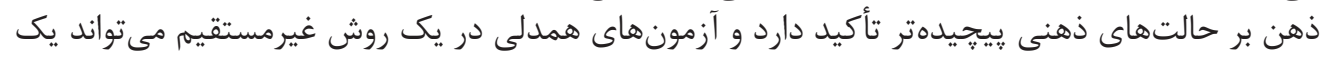
آزمون قابل اطمينانتر باشد. ذالتهيد

كليد وازهها:

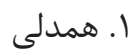

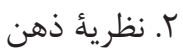

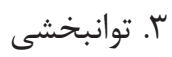

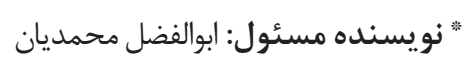
آدرس الكترونيكى: abolfazl796@gmail.com 


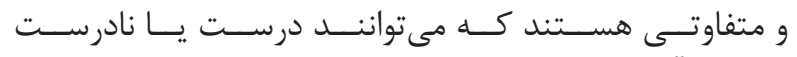

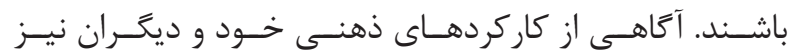

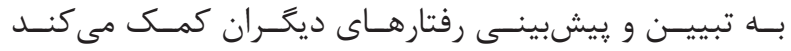

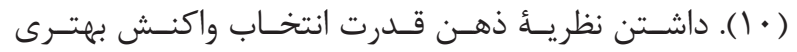

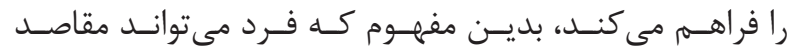

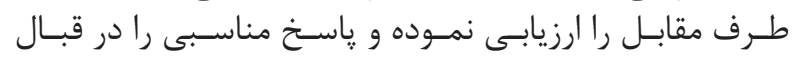

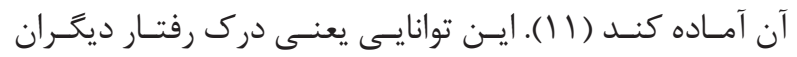

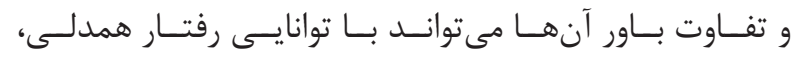

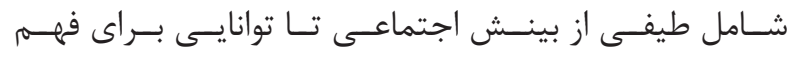

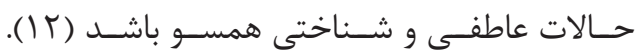

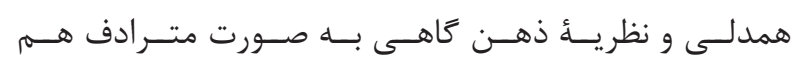

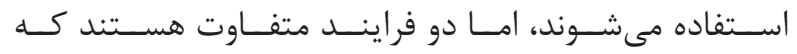

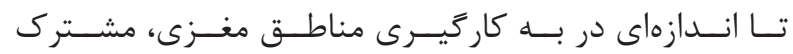

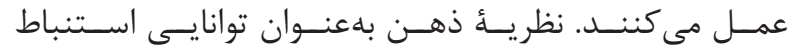

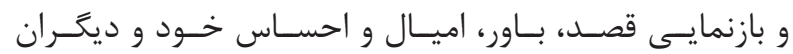

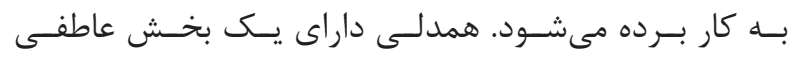

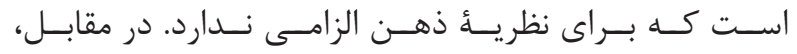

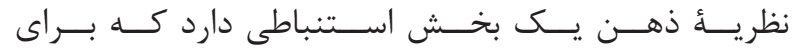

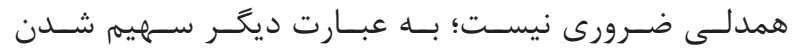

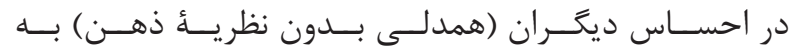

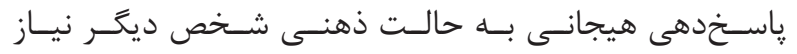

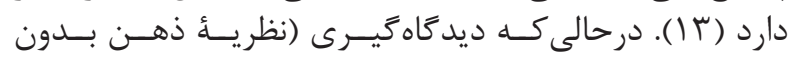

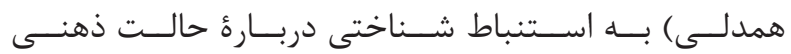

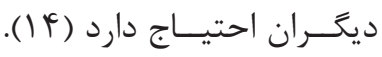

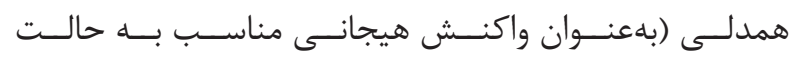

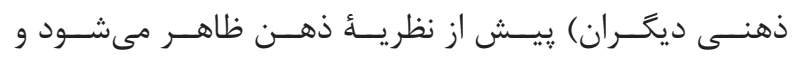

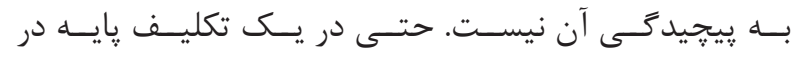

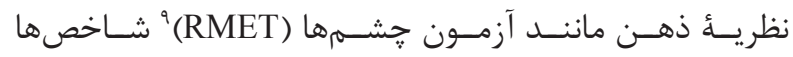

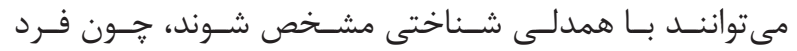

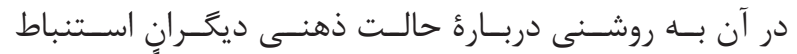

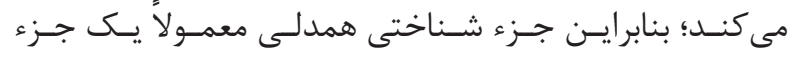

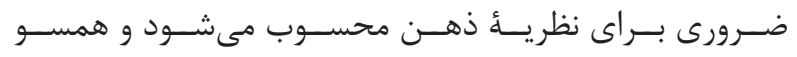

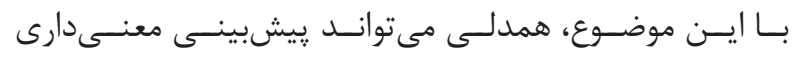

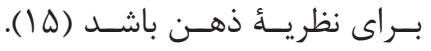
توانايسى نظريـــ ذهــن و همدلـى در افـراد مختلـف، متفــاوت

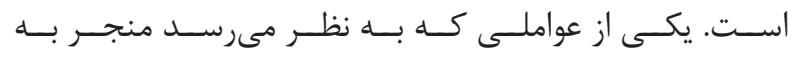

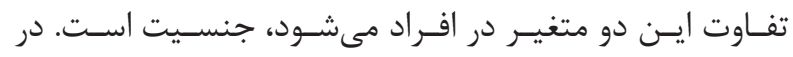

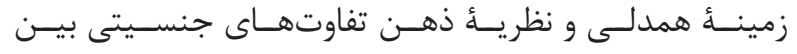

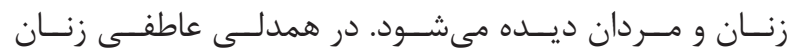

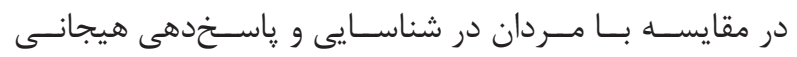

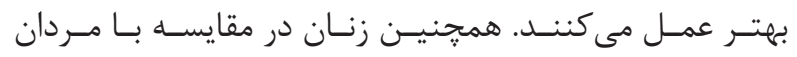

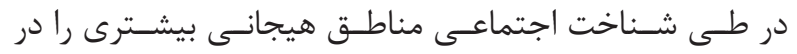

\footnotetext{
${ }^{1}$ Empathy

${ }^{2}$ Mirror neurons

${ }^{3}$ Limbic system

${ }^{4}$ Prefrontal

${ }^{5}$ Temporal
}

مقدمه

همدلـى ' موجـب افزايـش ظرفيـت بنياديـن افـراد در تنظيـهم

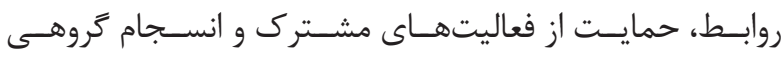

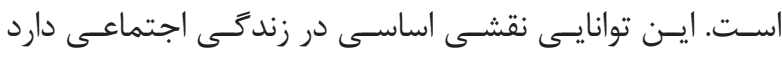

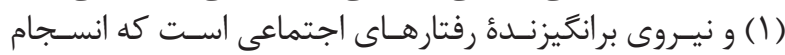

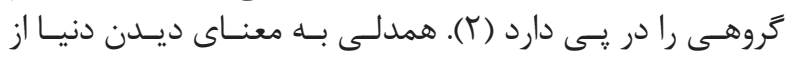

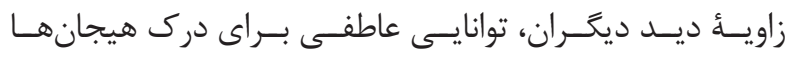

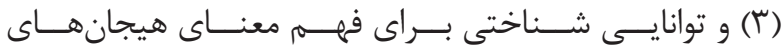

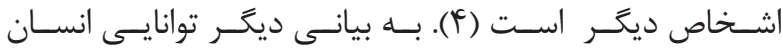

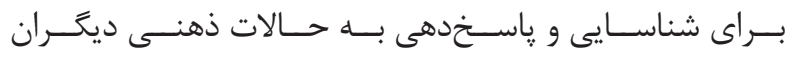

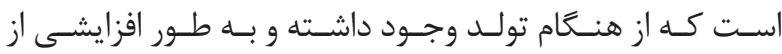

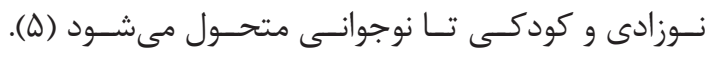

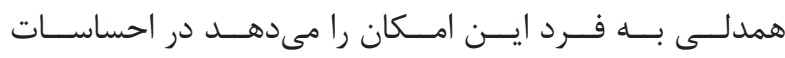

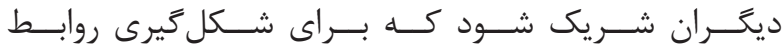

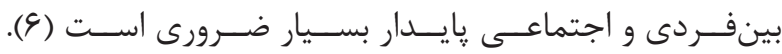

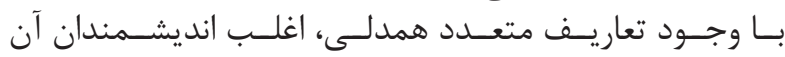

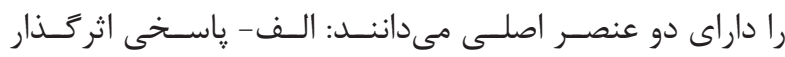

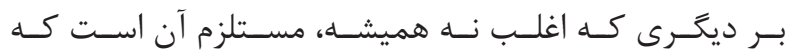

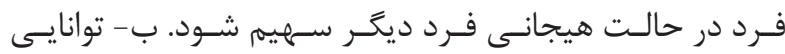

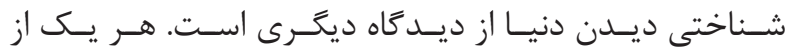

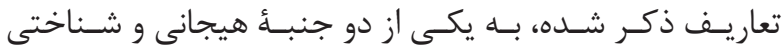

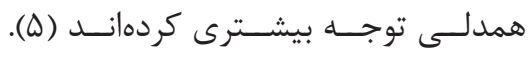

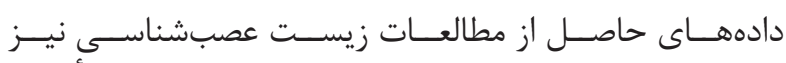

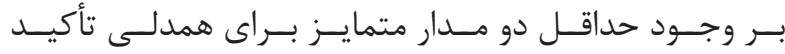

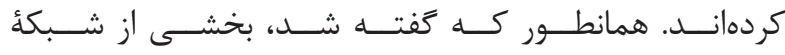

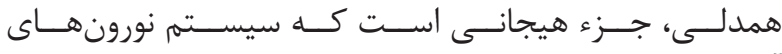

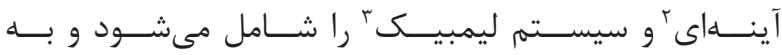

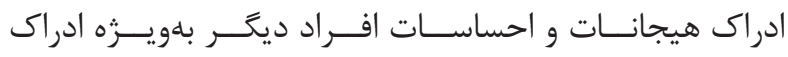

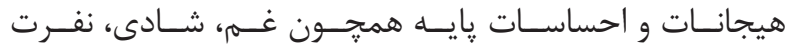

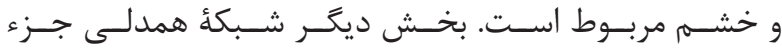

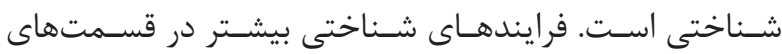

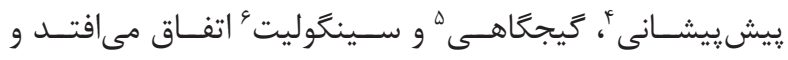

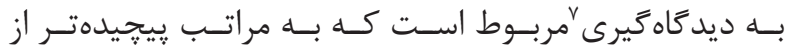

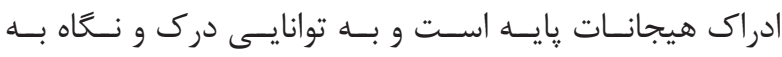

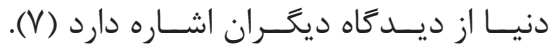

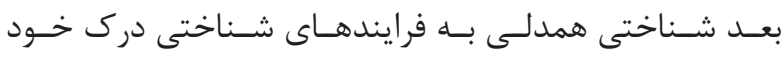

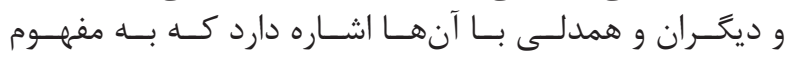

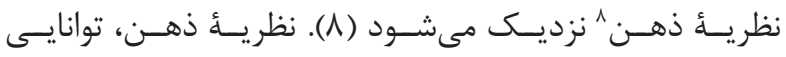

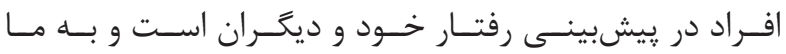

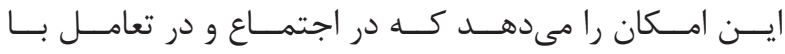

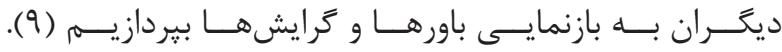

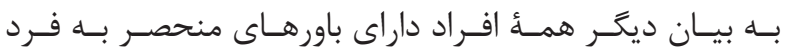

\footnotetext{
${ }^{6}$ Cingulate

${ }^{7}$ Perspective Taking

${ }^{8}$ Theory of mind

${ }^{9}$ Reading the mind in the eyes test
} 


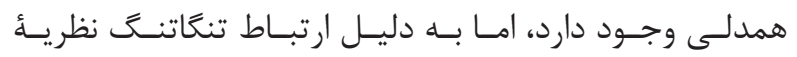

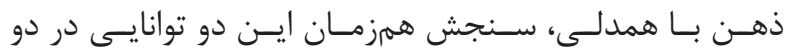

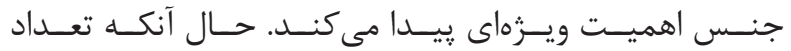

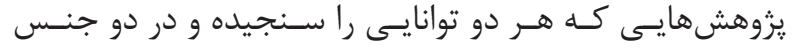

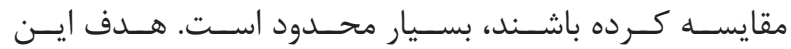

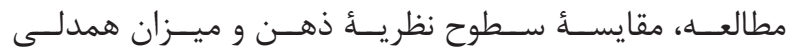

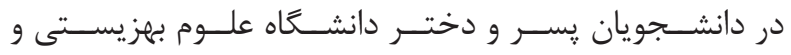

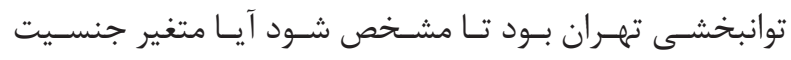

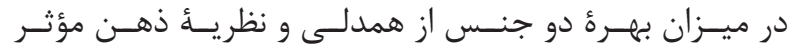

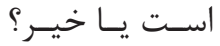

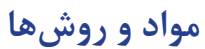

ايـن يزوهـش توصيفـى و از نــوع مطالعـات مقايسـهاى اسـت.

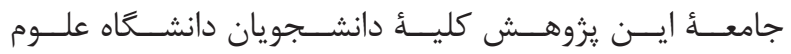

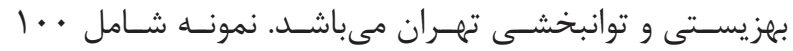

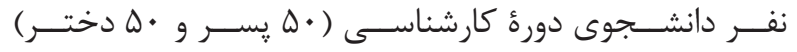

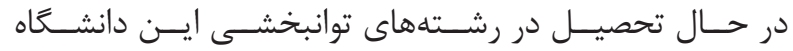

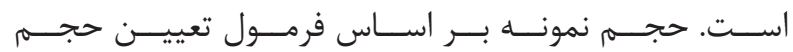

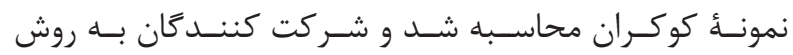

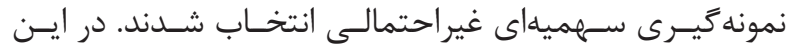

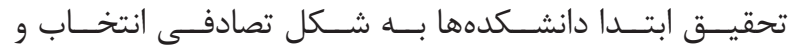

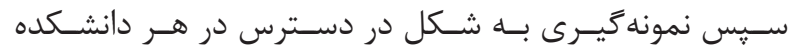

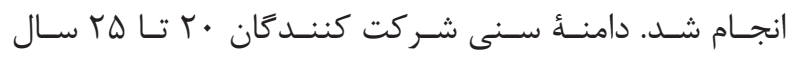

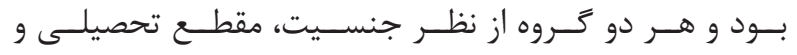

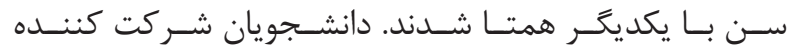

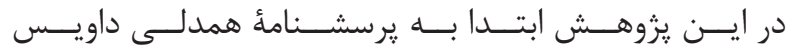
(DEQ)

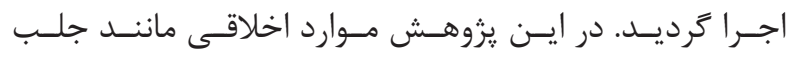

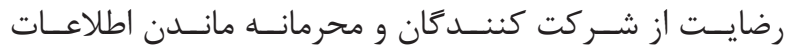

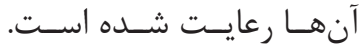

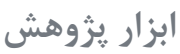

يرسشنامة همدلى داويس

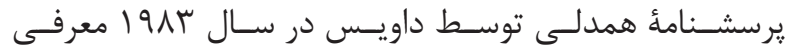

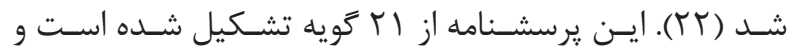

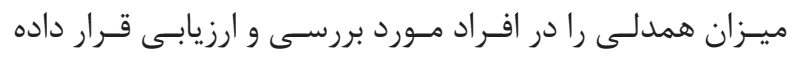

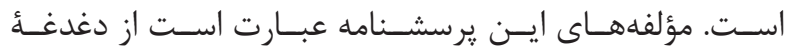

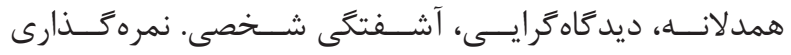

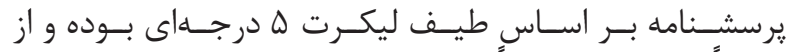

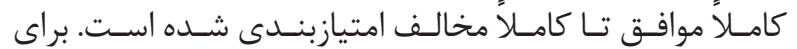

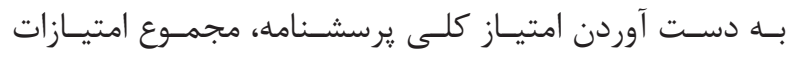

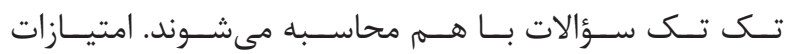

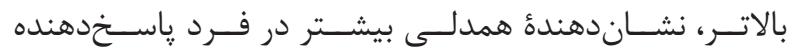

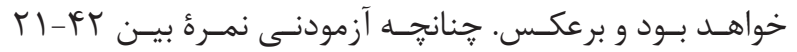

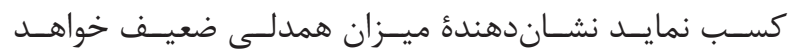

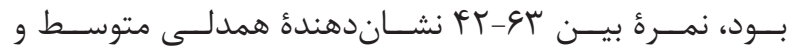

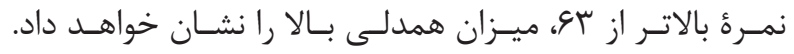

${ }^{10}$ Christov-Moore

${ }^{11}$ Adams

${ }^{12}$ Baron-Cohen

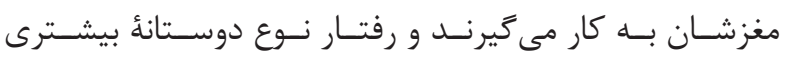

نشـان مى دهنــــ (1) (1).

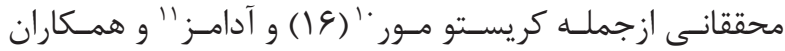

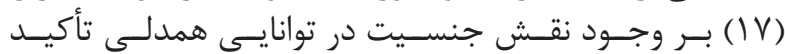

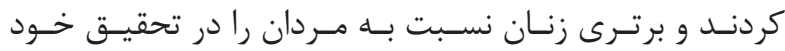

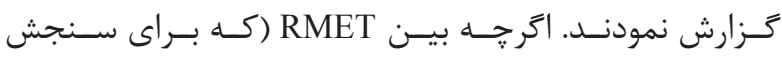

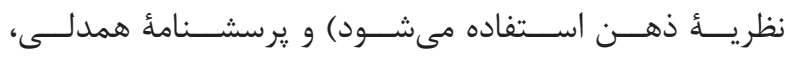

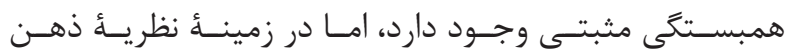

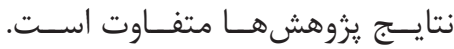

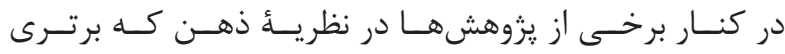

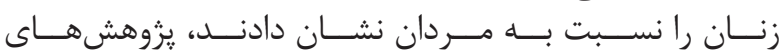

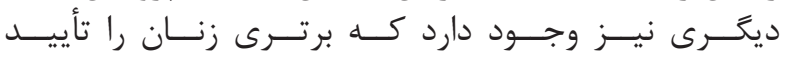

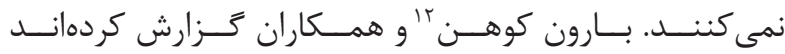

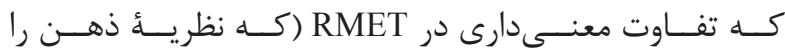

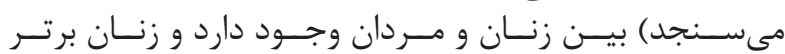

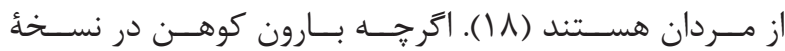

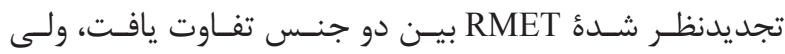

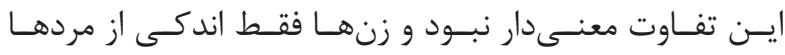

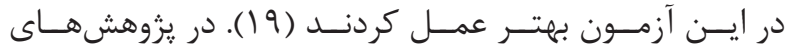

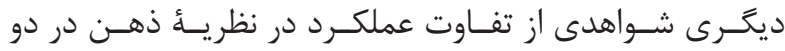

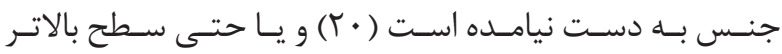

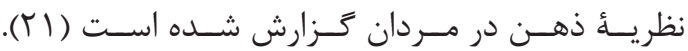

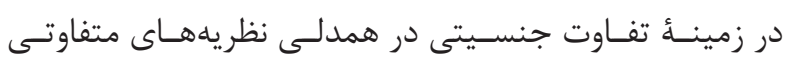

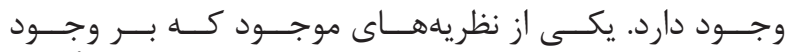

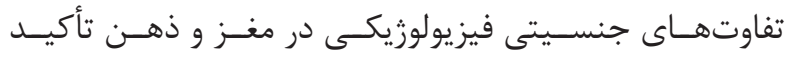

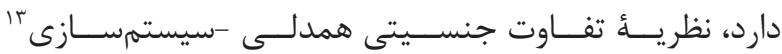

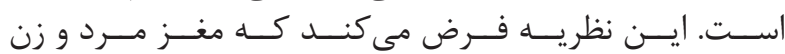

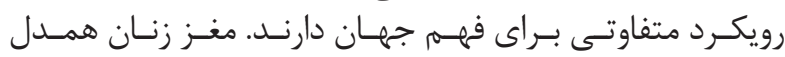

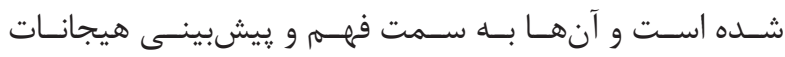

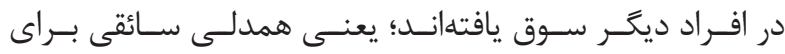

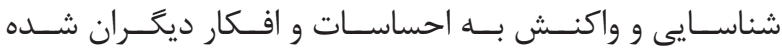

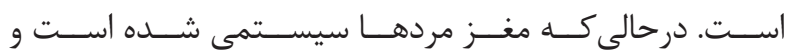

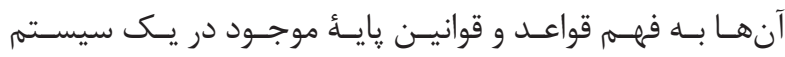

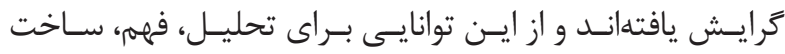

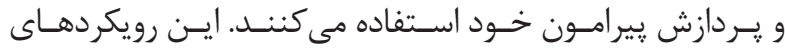

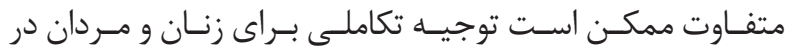

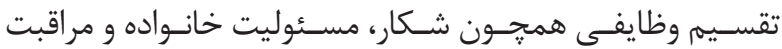

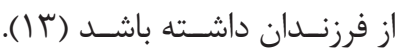

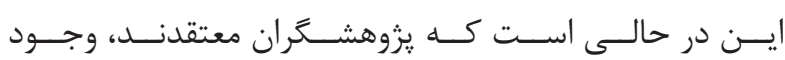

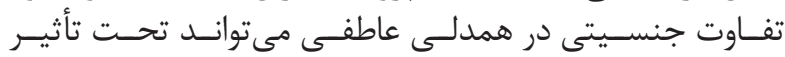

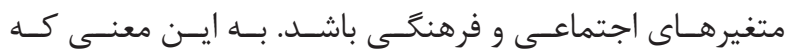

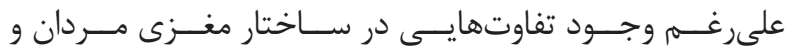

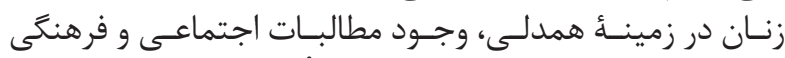

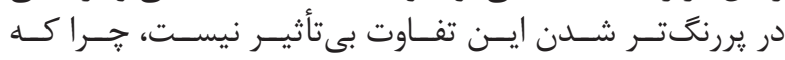

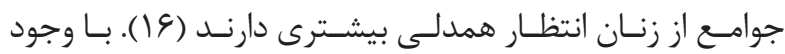

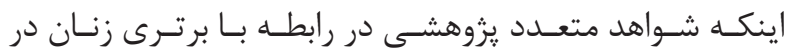

${ }^{13}$ Empathizing-systemizing (E-S) theory

${ }^{14}$ Davis empathy questionnaire 


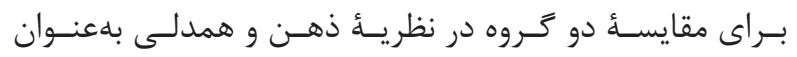

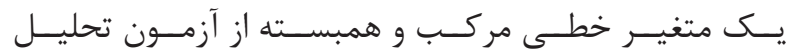

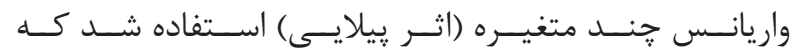

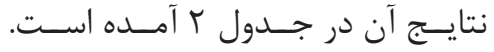

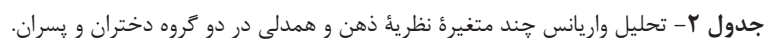
متغير

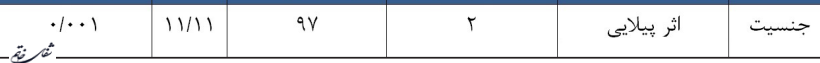

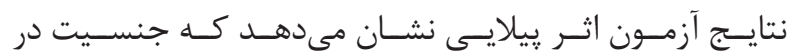

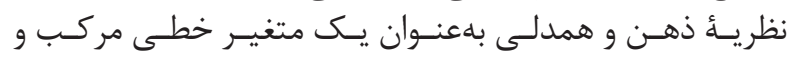

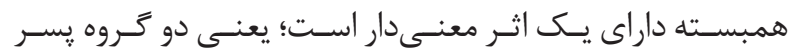

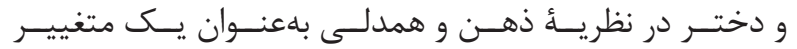

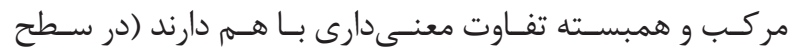

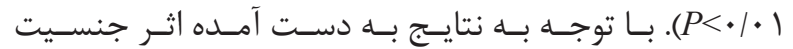

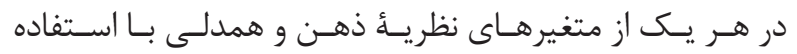

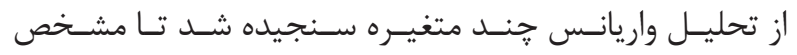

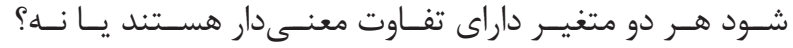

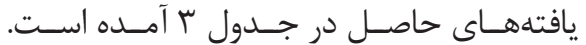

جدول ب- تحليل واريانس جِند متغيرءٔ اثر جنسيت در هر يك از متغيرهاى نظريئ ذهن و همدلى.

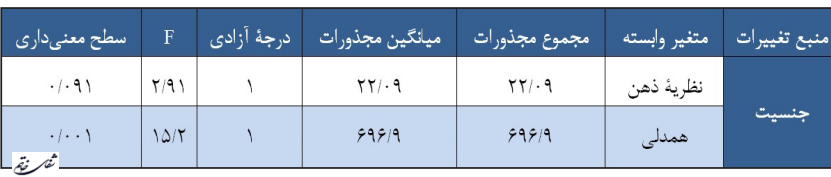

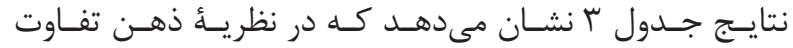

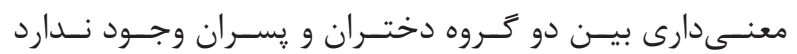

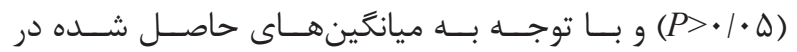

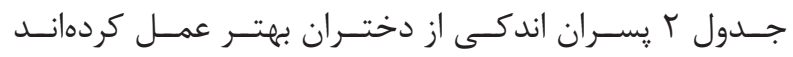

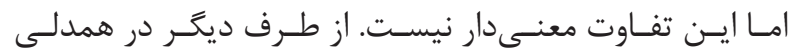

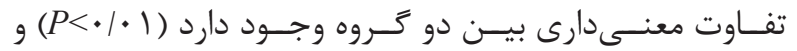

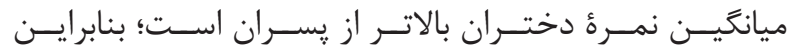

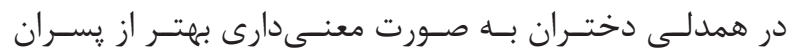

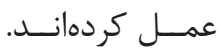

بحث و نتيجه

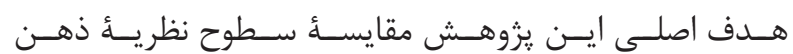

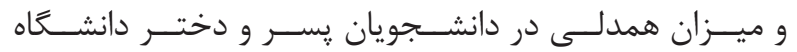

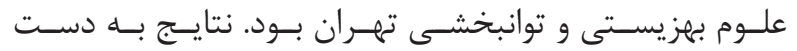

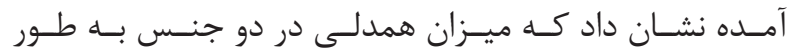

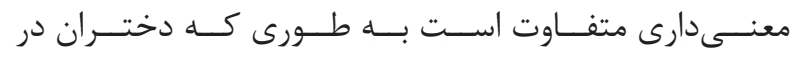

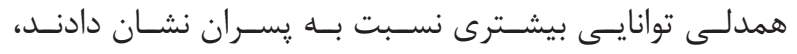

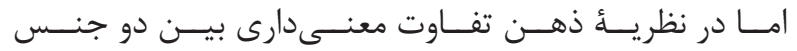

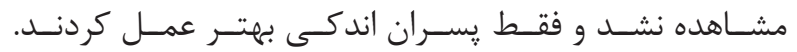

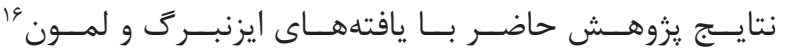

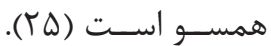

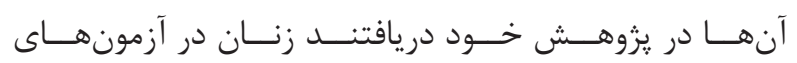

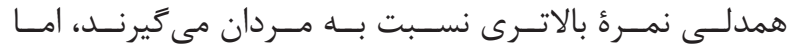

${ }^{15}$ Multivariable analyze of variance

${ }^{16}$ Eisenberg and Lemon

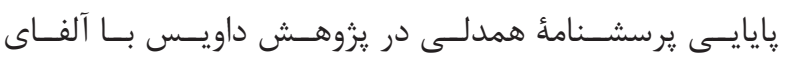

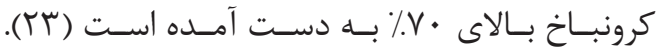

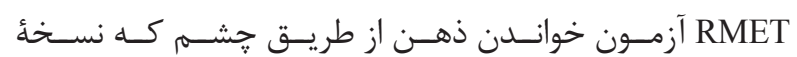

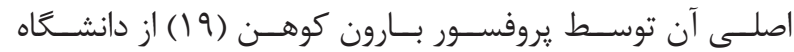

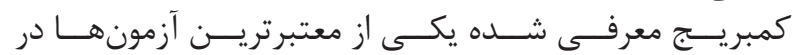

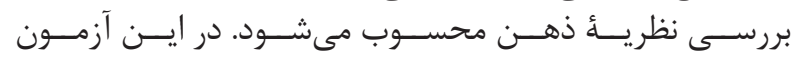

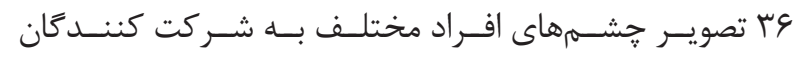

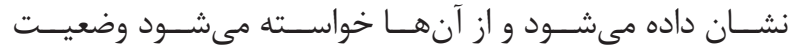

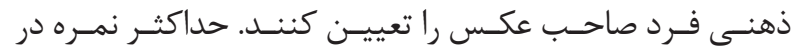

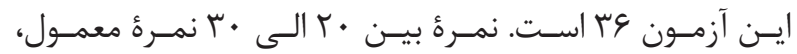

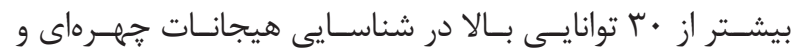

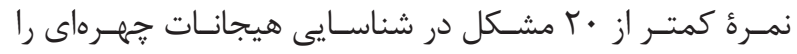

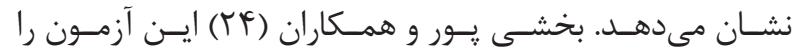

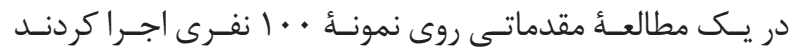

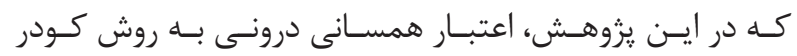

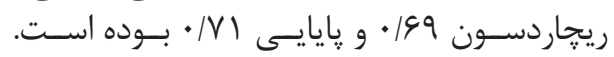

\section{تجزيه و تحليل آمارى}

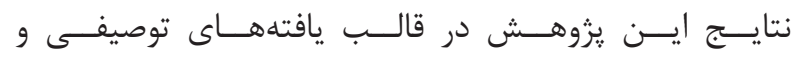

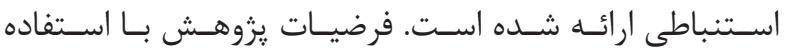

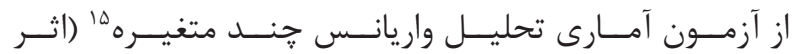

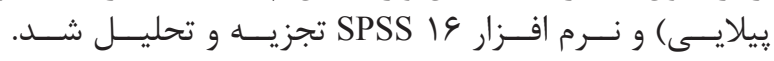

\section{بافتهها}

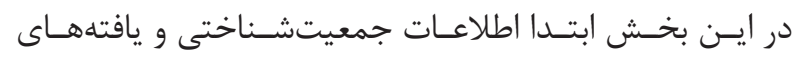

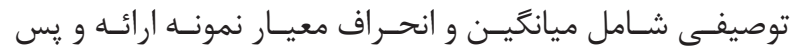

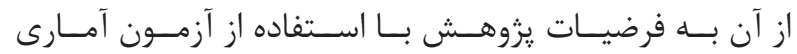

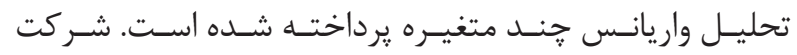

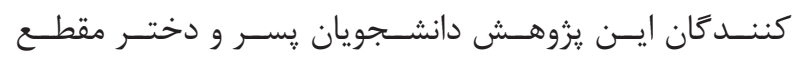

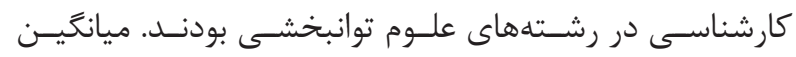

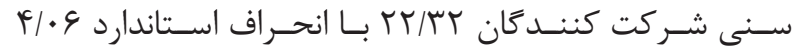

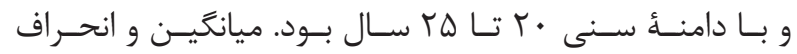

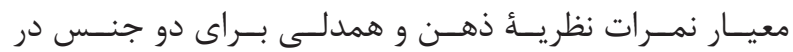

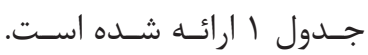

جدول ا- ميانكَين و انحراف معيار نمرههاى نظريئ ذهن و همدلى در شركت كندكًان دختر

\begin{tabular}{|c|c|c|c|}
\hline انحراف معيار & ميانگين & جنسيت & مثغير \\
\hline r/Dr & $r \cdot / T r$ & زن & \\
\hline$r / 9 \Delta$ & $4 / / 1 /$ & مرد & نظريةٌ ذهن \\
\hline T/VD & $r \cdot 19 \mathrm{~V}$ & كل & \\
\hline V/IV & $V \Psi / \mathcal{C}$ & زن & \\
\hline g/Tr & $9 N / 19$ & مرد & همدلى \\
\hline W/T & $V \cdot / \Lambda$ & كل & \\
\hline
\end{tabular}




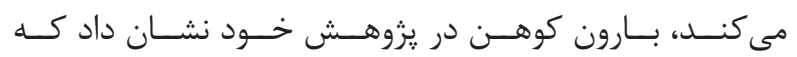

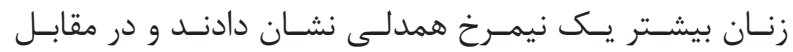

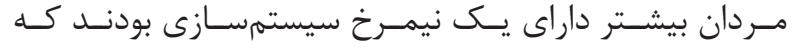

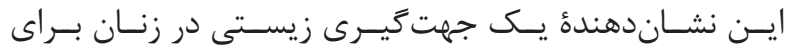

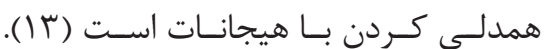

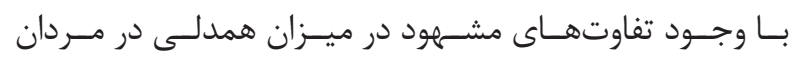

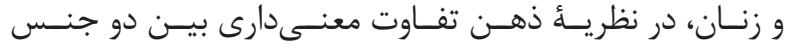

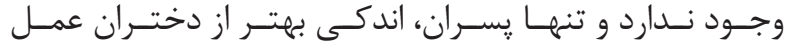

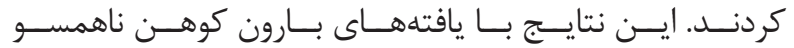

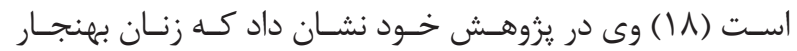

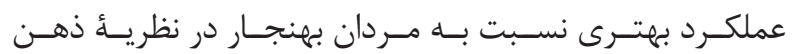

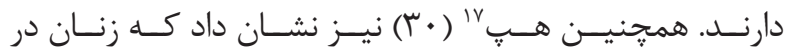

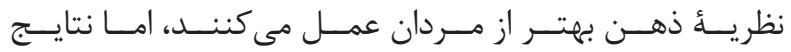

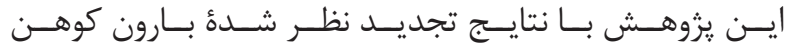

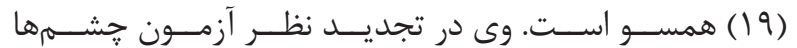

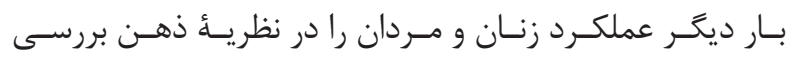

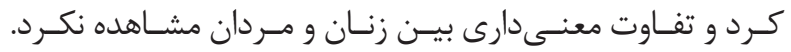

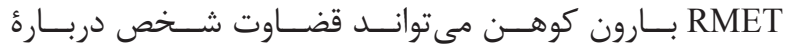

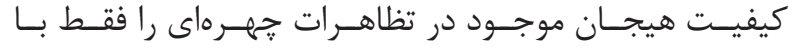

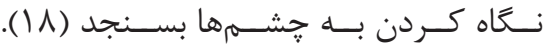

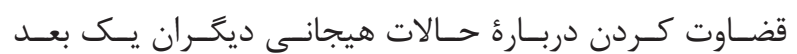

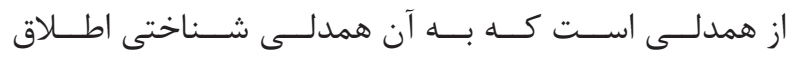

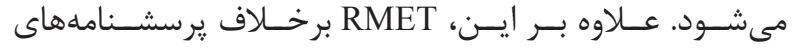

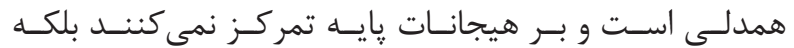

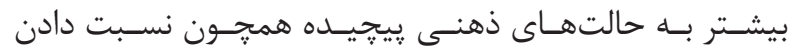

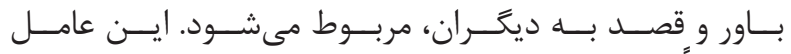

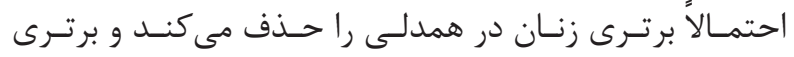

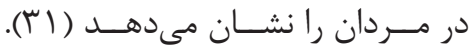

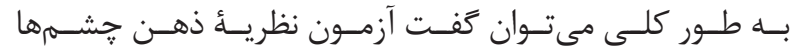

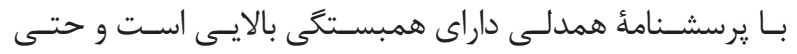

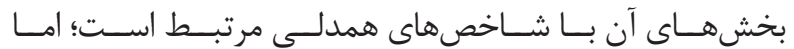

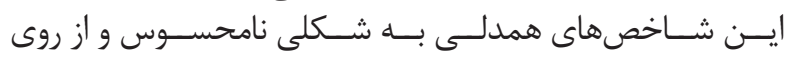

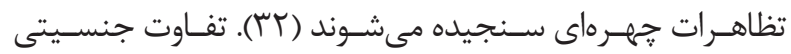

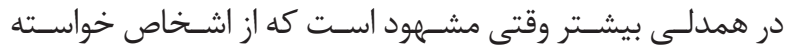

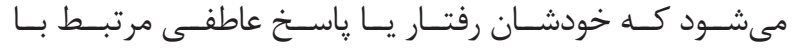

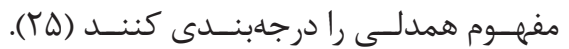

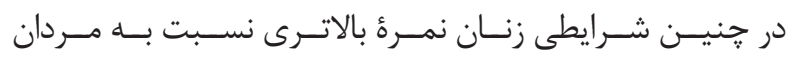

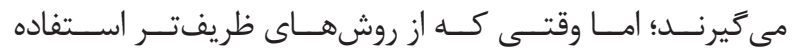

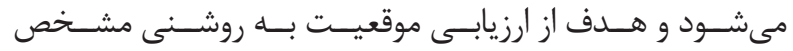

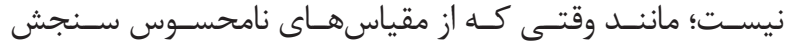

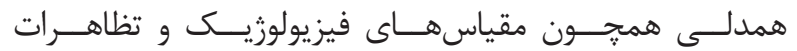

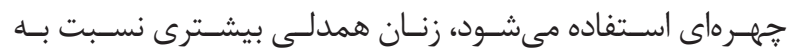

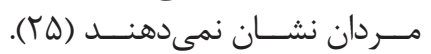

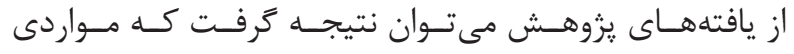

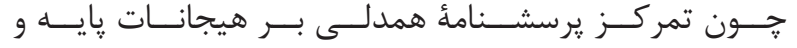
${ }^{17}$ Happé

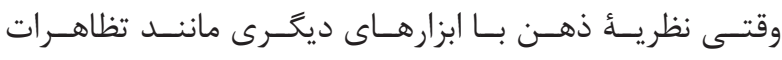

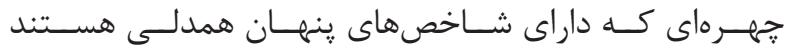

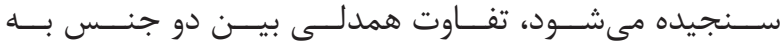

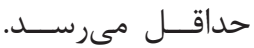

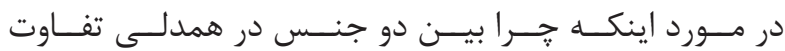

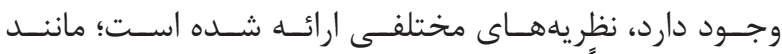

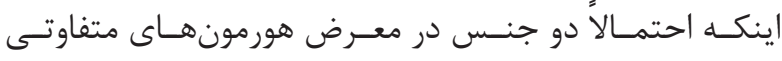

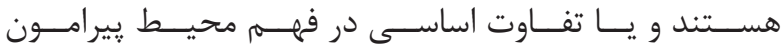

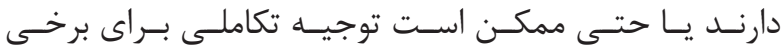

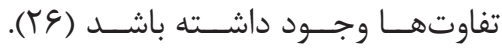

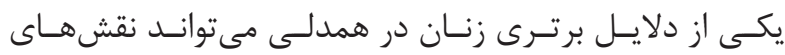

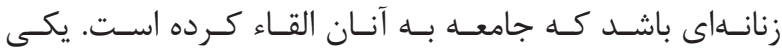

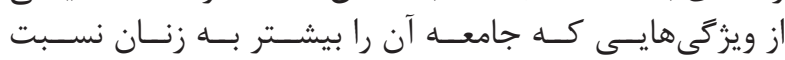

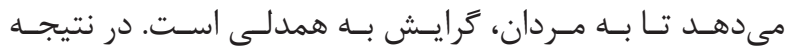

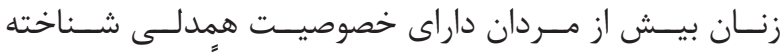

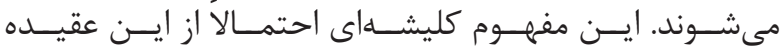

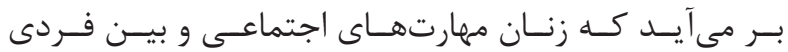

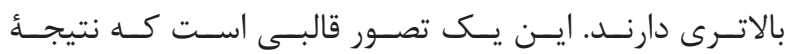

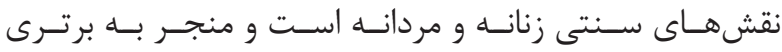

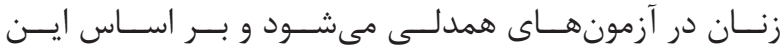

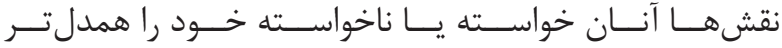

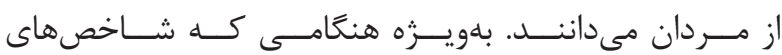

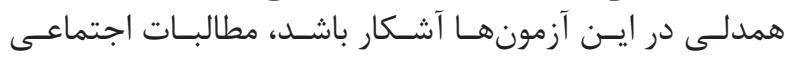

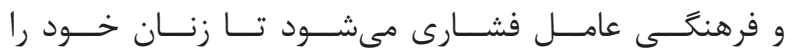

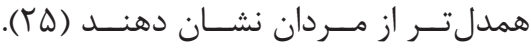

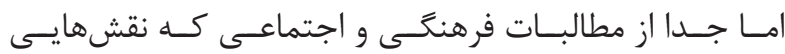

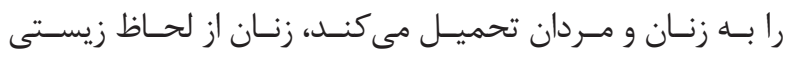

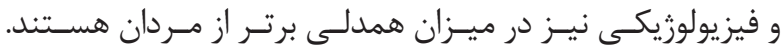

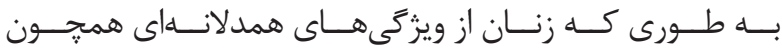

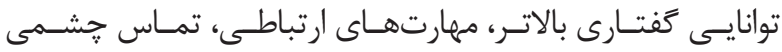

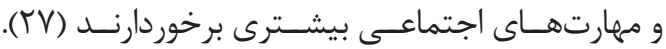

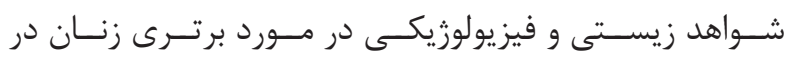

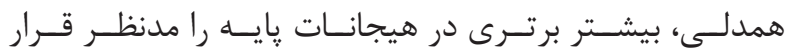

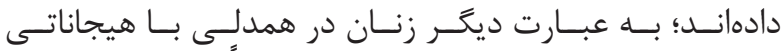

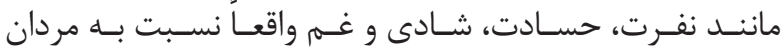

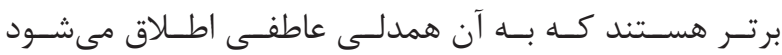

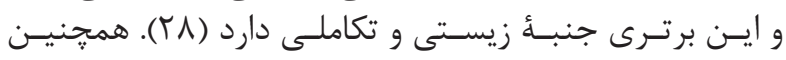

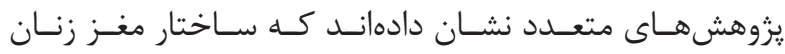

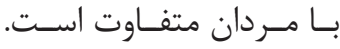

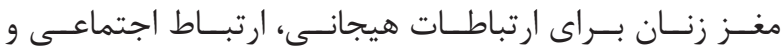

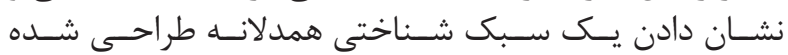

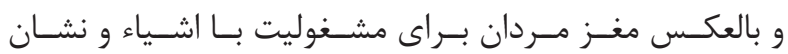

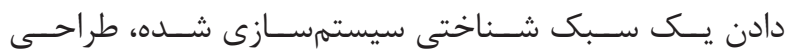

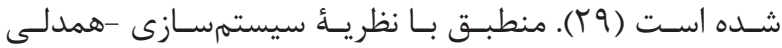

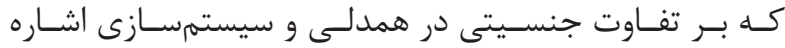




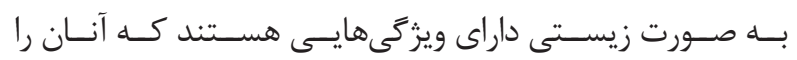

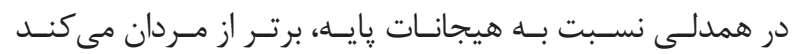

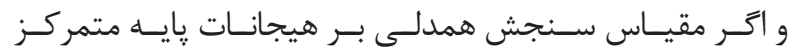

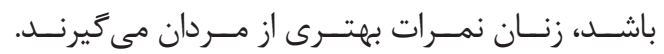

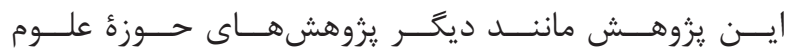

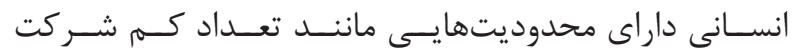

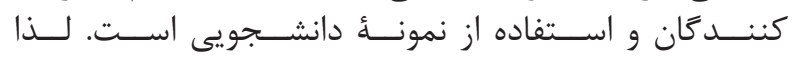

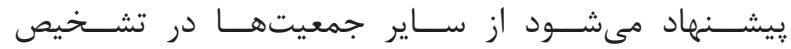

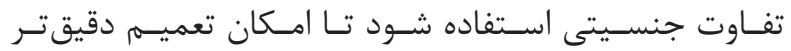

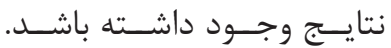

1. Rieffe C, Ketelaar L, Wiefferink CH. Assessing empathy in young children: construction and validation of an empathy questionnaire (EmQue). Pers Individ Dif. 2010; 49(5): 362-7.

2. Jolliffe D, Farrington DP. Examining the relationship between low empathy and bullying. Aggress Behav. 2006; 32(6): 540-50.

3. Bryant BK. An index of empathy for children and adolescents. Child Dev. 1982; 53(2): 413-25.

4. Hawkins KA, Trobst KK. Frontal lobe dysfunction and aggression: conceptual issues and research findings. Aggress Violent Behav. 2000; 5(2): 147-57.

5. Roth-Hanania R, Busch-Rossnagel N, HigginsD'Alessandro A. Development of self and empathy in early infancy: implications for atypical development. Infants Young Child. 2000; 13(1): 1-14.

6. HashemiT, Khalilzad Behrouzian S, Mashinchi Abbasi N. Gender, Comparison of Alexithymia and Theory of Mind in University StudentsSocial Cognition. 2013; 1(2): 6-13.

7. Zaki J, Ochsner KN. The neuroscience of empathy: progress, pitfalls and promise. Nat Neurosci. 2012; 15(5): $675-80$.

8. Flavell JH, Flavell ER, Green FL. Development of the appearance-reality distinction. Cogn Psych. 1983; 15(1): 95-120.

9. Perner J, Lang B, Stummer S. Theory of mind and executive function: Which depends on which. Unpublished manuscript. University of Salzburg. 1998.

10. Drury VM, Robinson E, Birchwood M. Theory of

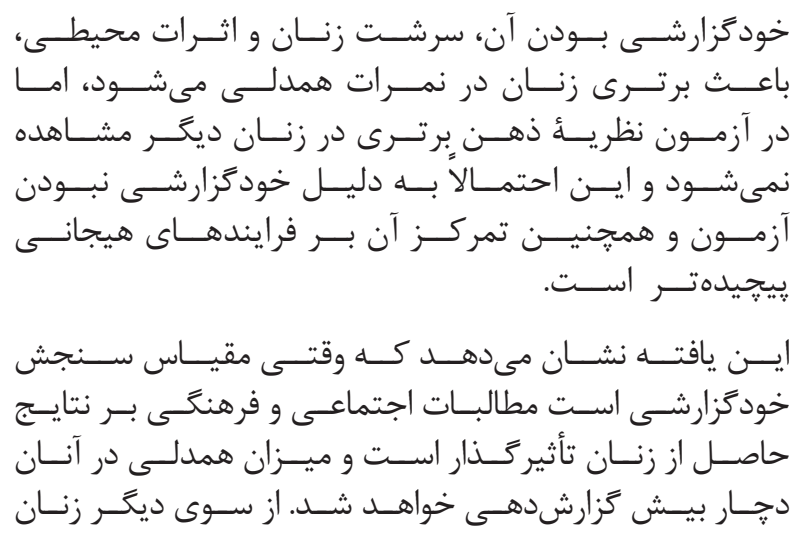

منابع

mind'skills during an acute episode of psychosis and following recovery. Psychol. Med. 1998; 28(05): 1101-12.

11. Krach S, Blümel I, Marjoram D, Lataster T, Krabbendam L, Weber J, et al. Are women better mindreaders? sex differences in neural correlates of mentalizing detected with functional MRI. BMC Neurosci. 2009; 10(1): 9. doi: 10.1186/1471-2202-10-9.

12. Eisenberg N, Fabes RA, Schaller M, Carlo G, Miller PA. The relations of parental characteristics and practices to children's vicarious emotional responding. Child Dev. 1991; 62(6): 1393-408.

13. Baron-Cohen S. Autism: the empathizingsystemizing (E-S) theory. J Cogn Neurosci. 2009; 1156(1): 68-80.

14. Singer T. The neuronal basis and ontogeny of empathy and mind reading: review of literature and implications for future research. Neurosci Biobehav Rev. 2006; 30(6): 855-63.

15. Korkmaz B. Theory of mind and neurodevelopmental disorders of childhood. Pediatr Res. 2011; 69: 101R-8R.

16. Christov-Moore L, Simpson EA, Coudé G, Grigaityte K, Iacoboni M, Ferrari PF. Empathy: gender effects in brain and behavior. N Neurosci Biobehav Rev. 2014; 46: 604-27.

17. Adams GR, Schvaneveldt JD, Jenson GO. Sex, age and perceived competency as correlates of empathic ability in adolescence. Adolescence. 1979; 14(56): 811-8.

18. Baron-Cohen S, Jolliffe T, Mortimore C, Robertson M. Another advanced test of theory of mind: evidence 
from very high functioning adults with autism or asperger syndrome. J. Child Psychol. Psychiatry. 1997; 38(7): 813-22.

19. Baron-Cohen S, Wheelwright S, Hill J, Raste Y, Plumb I. The "reading the mind in the eyes" test revised version: a study with normal adults, and adults with asperger syndrome or high-functioning autism. J. Child Psychol. Psychiatry. 2001; 42(2): 241-51.

20. Jarrold C, Butler DW, Cottington EM, Jimenez F. Linking theory of mind and central coherence bias in autism and in the general population. Dev. Psychol. 2000; 36(1): 126.

21. Russell TA, Tchanturia K, Rahman Q, Schmidt U. Sex differences in theory of mind: a male advantage on Happé's “cartoon” task. Cogn Emot. 2007; 21(7): 1554-64.

22. Davis MH. Empathic concern and the muscular dystrophy telethon empathy as a multidimensional construct. Pers Soc Psychol Bull. 1983; 9(2): 223-9.

23. Davis MH. Measuring individual differences in empathy: evidence for a multidimensional approach. J Pers Soc Psychol. 1983; 44(1): 113-26.

24. Bakhshipor AMA, M.Kanjani, Z.Ranjbar, F.Bafande, H. Theory of mind deficits in schizophrenic patients with positive and negative symptoms: a case defect or trait. Journal of Tabriz University of Medical Sciences. 2011; 33(6): 32-8.

25. Eisenberg N, Lennon R. Sex differences in empathy and related capacities. Psychol. Bull. 1983; 94(1): 100-31.

26. McClure I. The essential difference: men, women and the extreme male brain. BMJ. 2003; 327(7405): 57.

27. Baron-Cohen S, Lutchmaya S, Knickmeyer R. Prenatal testosterone in mind: amniotic fluid studies. MIT Press. 2004.

28. Aleman A, Swart M. Sex differences in neural activation to facial expressions denoting contempt and disgust. PloS One. 2008; 3(11): e3622. doi: 10.1371 /journal.pone. 0003622 .

29. Ibanez A, Huepe D, Gempp R, Gutiérrez V, Rivera-Rei A, Toledo MI. Empathy, sex and fluid intelligence as predictors of theory of mind. Pers Individ Dif. 2013; 54(5): 616-21.

30. Happé FG. An advanced test of theory of mind: understanding of story characters' thoughts and feelings by able autistic, mentally handicapped, and normal children and adults. J Autism Dev Disord. 1994; 24(2): 129-54.

31. Williams LM, Mathersul D, Palmer DM, Gur RC, Gur RE, Gordon E. Explicit identification and implicit recognition of facial emotions: I. age effects in males and females across 10 decades. J Clin Exp Neuropsychol. 2009; 31(3): 257-77.

32. Dressler GR. The cellular basis of kidney development. Annu Rev Cell Dev Biol. 2006; 22: 509-29. 